\title{
Observation of Electronic Structure Minima in High-Harmonic Generation
}

\author{
Hans Jakob Wörner, ${ }^{1}$ Hiromichi Niikura, ${ }^{1,2}$ Julien B. Bertrand, ${ }^{1,3}$ P. B. Corkum, ${ }^{1,3}$ and D. M. Villeneuve ${ }^{1}$ \\ ${ }^{1}$ National Research Council of Canada, 100 Sussex Drive, Ottawa, Ontario, Canada K1A OR6 \\ ${ }^{2}$ PRESTO, Japan Science and Technology Agency, Sanbancho building, 5-Sanbancho, Chiyodaku, Tokyo 102-0075, Japan \\ ${ }^{3}$ Department of Physics, University of Ottawa, 150 Louis Pasteur, Ottawa, Ontario, Canada K1N 6N5 \\ (Received 27 October 2008; revised manuscript received 17 December 2008; published 10 March 2009)
}

We report detailed measurements of the high-harmonic spectra generated from argon atoms. The spectra exhibit a deep minimum that is shown to be independent of the laser intensity, and is thus a clear measure of the electronic structure of the atom. We show that exact field-free continuum wave functions reproduce the minimum, but plane wave and Coulomb wave functions do not. This remarkable observation suggests that electronic structure can be accurately determined in high-harmonic experiments despite the presence of the strong laser field. Our results clarify the relation between high-harmonic generation and photoelectron spectroscopy. The use of exact continuum functions also resolves the ambiguity associated with the choice of the dispersion relation.

DOI: 10.1103/PhysRevLett.102.103901

PACS numbers: 42.65.Ky, 32.80.Wr, 42.65.Re

In recent years, high-harmonic generation (HHG) has developed into the main tool of attosecond science. It has permitted the production of attosecond pulses [1], the observation of molecular dynamics [2], and the imaging of molecular orbitals with angstrom resolution [3]. HHG is most simply understood in terms of the three-step model: a molecule exposed to a strong laser field is tunnel ionized, the liberated electron is then accelerated by the field oscillations which can force it to recollide with the parent ion [4]. Recollision is the common step of most strong-field processes that permit the observation of dynamical events with attosecond temporal and angstrom spatial resolution [5]. When the continuum electron wave packet recombines with the ion core of a diatomic molecule, an interference takes place that is characteristic of the internuclear separation of the molecule. The phenomenon has been discovered in calculations of the $\mathrm{HHG}$ spectrum of $\mathrm{H}_{2}^{+}$[6] and it has been invoked to explain minima observed in the HHG spectrum of aligned $\mathrm{CO}_{2}$ molecules [7,8]. Here we report the observation and interpretation of a new kind of minimum in HHG that results solely from the electronic structure of the target.

The current understanding of processes occurring in a strong laser field relies on the strong-field approximation, but its shortcomings for quantitative modeling are now becoming apparent. When the electron is far away from the ion core, its motion is dominated by the laser field and therefore its wave function is well represented by plane wave functions or the time-dependent Volkov functions. The difficulty of the coupled Coulomb-Volkov problem is usually addressed by including the ionic potential in the eikonal approximation [9]. We follow a different strategy, treating the ionic potential exactly and neglecting the effect of the laser field during recombination.

In this Letter we show a prominent minimum observed in HHG in argon atoms. We show that the position of the minimum is not influenced by the strong laser field that is present during the recombination process. This remarkable observation implies that high-harmonic experiments can directly measure the field-free electronic structure of the target atom or molecule. Experimentally, we study the argon atom as one of the most frequently used in highharmonic and attosecond pulse generation and we show that the position of the minimum is independent of intensity and driving wavelength. Theoretically, we develop a method to calculate high-harmonic spectra from field-free continuum functions of an effective potential. The calculation of recombination cross sections for HHG using scattering functions has been introduced in Ref. [10]. This approach has also been used to predict harmonic spectra of the rare gas atoms [11] and $\mathrm{H}_{2}^{+}$[12]. We show that the minimum observed in HHG in argon atoms lies close to the minimum in the photoionization spectrum that is known as Cooper minimum [13]. Indeed, the last step of HHG is similar to photorecombination of an ion and an electron. We show that our method is capable of quantitatively reproducing the position of the observed minimum in contrast to other methods using plane waves or Coulomb waves.

The experimental setup consists of a chirped-pulse amplification Ti:sapphire laser system (KM Labs), a hollowcore fiber setup for pulse compression, and a vacuum chamber for generation and characterization of highharmonic radiation. The laser system provides pulses of $2 \mathrm{~mJ}$ energy and $35 \mathrm{fs}$ duration at a repetition rate of $1 \mathrm{kHz}$. The pulses are focused into a hollow-core fiber filled with argon to achieve self-phase modulation [14] and are subsequently compressed using chirped mirrors. The typical duration of the compressed pulses was $6-8$ fs. These pulses are focused $\sim 1 \mathrm{~mm}$ below the orifice of a pulsed nozzle generating a supersonic expansion of argon. This setup minimizes the effects of phase mismatch and reabsorption 
of the high-harmonic radiation and leads to the observation of the single-atom response. The generated high-order harmonics are sent into an extreme ultraviolet spectrometer consisting of an aberration-corrected concave grating that spectrally disperses the harmonics and images them onto a microchannel plate detector. A CCD camera accumulates the spectral image and transfers it to a computer. The image is integrated over the spatial dimension to provide the high-harmonic spectrum.

High-harmonic spectra generated in argon using an 8 fs laser pulse centered at $780 \mathrm{~nm}$ at different intensities are shown in Fig. 1. In the region of low photon energies, the spectrum shows the usual progression of odd harmonics of the fundamental wavelength which turns into a continuum close to the cutoff. The most striking feature of the spectrum is the presence of a deep minimum around harmonic 33.

Different origins are conceivable for minima in highharmonic spectra. Minima can originate from the structure of the atom or molecule. Their position is approximately independent of the laser intensity as is the case in twocenter interference [6]. A minimum can also originate from dynamics of either the electron in the continuum or of the molecular cation following tunnel ionization and/or the action of the strong laser field. An example of continuum dynamics has been reported using a mixture of helium and neon as nonlinear medium [15]. Such minima are associ-

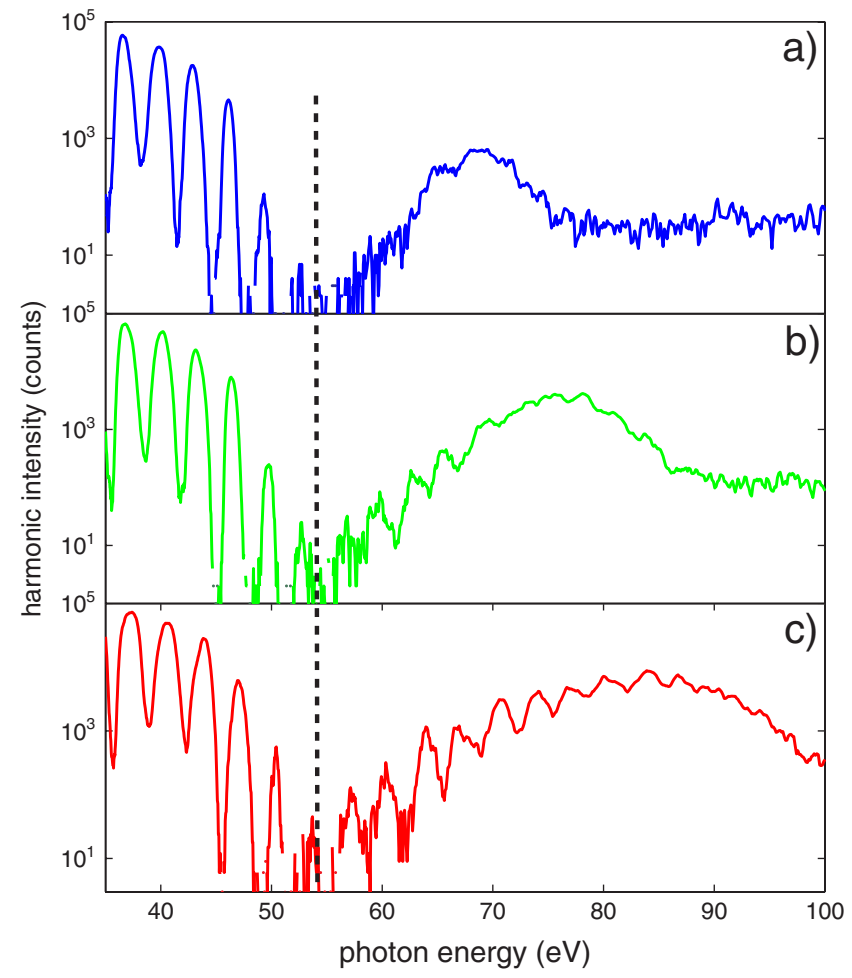

FIG. 1 (color online). High-harmonic spectra generated in argon using an 8 fs laser pulse centered at $780 \mathrm{~nm}$. The three different panels correspond to the laser intensities $2.5 \times$ $10^{14} \mathrm{~W} / \mathrm{cm}^{2} \quad$ (a), $2.9 \times 10^{14} \mathrm{~W} / \mathrm{cm}^{2} \quad$ (b), and $3.5 \times$ $10^{14} \mathrm{~W} / \mathrm{cm}^{2}$ (c). ated with a well-defined recollision time and their location in photon energy (i.e., harmonic order) would therefore shift approximately linearly with intensity. It would also depend strongly on the wavelength of the fundamental field.

With increasing intensities the cutoff in the highharmonic spectra shown in Fig. 1 extends clearly, but the position of the minimum is left unchanged. Measurements at longer wavelengths have revealed a minimum at the same photon energies as reported here [16]. This proves that the minimum observed in high-harmonic spectra of argon is associated with the electronic structure of the atom rather than with its dynamics in the laser field and is independent of the laser source.

We find that the minimum in argon appears at $53 \pm$ $3 \mathrm{eV}$. This is close to the observed position of the Cooper minimum in the single-photon-ionization efficiency [17]. The minimum in argon has been reported many times in the literature since the early days of HHG [18]. The minimum is much deeper in our spectra because we use an ultrashort pulse that extends the cutoff and places the minimum into the plateau.

We now show that we can accurately model the recombination step in HHG using field-free effective oneelectron procedures commonly used in photoionization spectroscopy. The potential is used to generate both the bound (ground) state and the continuum wave functions, neglecting the laser field at the moment of recombination. Without the laser field, the problem is spherically symmetric. The electronic wave function is represented as a product

$$
\Psi(r, \theta, \phi)=\frac{1}{r} \phi_{\ell}(r) Y_{\ell}^{m}(\theta, \phi)
$$

of a reduced radial wave function $\phi_{\ell}(r)$ and spherical harmonics. $\ell$ stands for the angular momentum quantum number of the electron and $m$ its projection quantum number. An effective one-electron potential [19]

$$
V_{\ell}(r)=\frac{\ell(\ell+1)}{2 r^{2}}-\frac{1}{r}-\frac{A e^{-r}+(17-A) e^{-C r}}{r}
$$

with $(A=5.4, C=3.682)$ is used and spin-orbit interaction is neglected. The ground state wave function $(3 p)$ is generated by diagonalizing the Hamiltonian for $\ell=1$ on a numerical grid. The continuum functions are generated by numerically integrating the Schrödinger equation for a given (positive) kinetic energy of the electron using the Numerov algorithm [20]. The normalization is performed at asymptotically large radial coordinates.

Figure 2 represents the bound and continuum wave functions for argon. The two-dimensional cut through the continuum wave function was obtained by summing all partial wave components from $\ell=0$ to $\ell=50$ that were obtained from numerical integration of the effective potential in Eq. (2). The continuum wave function consists of a plane wave propagating along the $x$ axis from left to right 


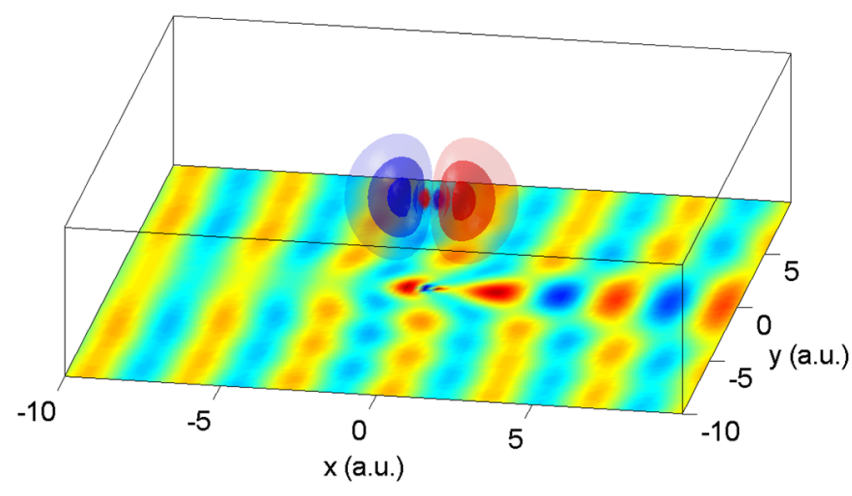

FIG. 2 (color online). $3 p_{x}\left(m_{\ell}=0\right)$ orbital wave function of argon and two-dimensional cut through the real part of the continuum wave function for $k=1.8$ a.u. The atomic orbital was obtained from a quantum chemical $a b$ initio calculation using the Hartree-Fock method. The two colors correspond to different signs of the wave function and their intensity of color codes the amplitude. The continuum wave function was calculated from the effective potential given in the text by adding the components $\ell=0-50$. The color code represents the amplitude.

and the superimposed scattered wave that strongly distorts the appearance of the plane wave.

As is the case in photorecombination, the continuum wave function consists of an incoming plane wave and a scattered spherical wave. The weights of the partial wave expansion are established as $c_{\ell}=i^{\ell} \sqrt{4 \pi(2 \ell+1)} e^{i \delta_{\ell}}$, where $\delta_{\ell}$ is the partial wave phase shift. The ground state wave function is represented by $\ell=1, m=0$ and the recombining portion of the continuum function has $\ell=0$ or $2, m=0$ by virtue of the symmetry properties of the dipole operator. The choice of $m=0$ for the bound state is motivated by the experimental evidence that tunnel ionization of a rare gas atom leads to orbital alignment of the ion that is created [21,22]. The recolliding electron wave packet generated by a linearly polarized laser pulse can thus only contain contributions from $m=0$ and the recombination leads to a dipole oscillation that is parallel to the laser polarization axis. The recombination dipole moment is therefore given by

$$
D \propto c_{0} a_{0}\left\langle\phi_{n, \ell_{g}}|r| \phi_{\epsilon, \ell=0}\right\rangle+c_{2} a_{2}\left\langle\phi_{n, \ell_{g}}|r| \phi_{\epsilon, \ell=2}\right\rangle .
$$

$\phi_{n, \ell_{g}}$ is the radial part of the bound ground state of principal and angular momentum quantum numbers $n$ and $\ell_{g}$, respectively. $\phi_{\epsilon, \ell}$ is the radial part of the continuum wave function of kinetic energy $\epsilon$ and angular momentum quantum number $\ell$, respectively. $a_{\ell}$ is the angular part of the integral given by $a_{\ell}=\left\langle Y_{\ell_{g}}^{m=0}|\cos \theta| Y_{\ell}^{m=0}\right\rangle$, where $Y_{\ell}^{m}$ represents the spherical harmonics.

Figure 3(a) shows the radial integrals $\left\langle\phi_{n, \ell_{g}=1}|r| \phi_{\epsilon, \ell}\right\rangle$ for $s$ and $d$ waves as a function of the emitted photon energy. Figure 3(b) shows the squared amplitude of the recombination dipole $|D|^{2}$ for different continuum functions. The radial integral for $d$-wave recombination changes sign around $46 \mathrm{eV}$. This causes a deep minimum

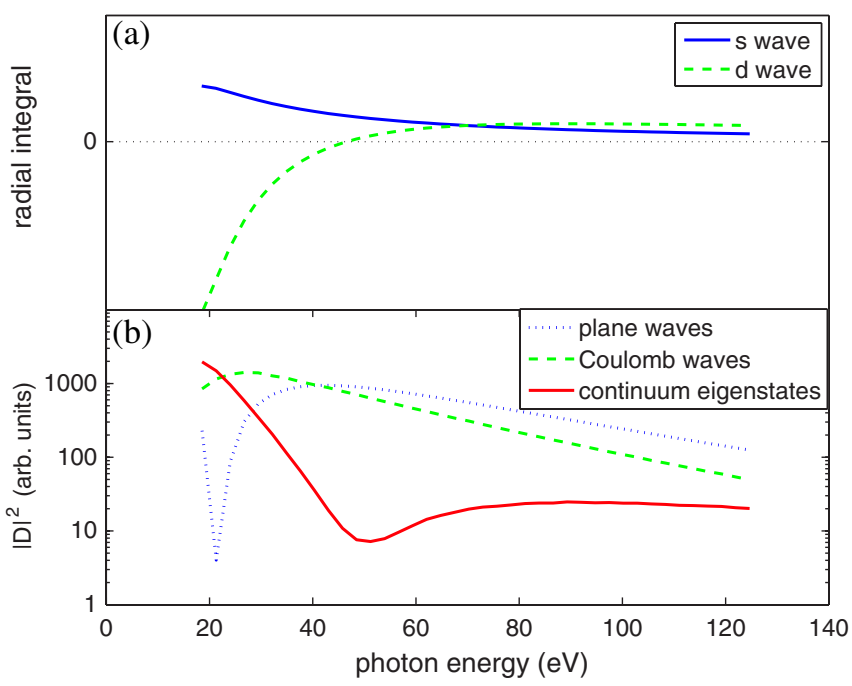

FIG. 3 (color online). (a) Radial integrals $\left\langle\phi_{3 p}|r| \phi_{\epsilon, \ell}\right\rangle$ for $s$ waves (full line), $d$ waves (dashed line) for argon as a function of the emitted photon energy. (b) Square modulus of the recombination dipoles $|D|^{2}$ as a function of the photon energy for argon. The full line is the result obtained with exact continuum wave functions, the dashed line was obtained from Coulomb wave functions, and the dotted line from plane wave functions. Only the position predicted by exact continuum functions agrees with the experimental result.

in the total recombination amplitude at $51 \mathrm{eV}$. In addition, it induces a variation of its phase by 2.6 rad over an interval of $20 \mathrm{eV}$ around this energy (not shown). The minimum predicted using plane wave functions is situated close to $21 \mathrm{eV}$. The calculation with Coulomb waves predicts no minimum above the ionization threshold.

The minimum observed in HHG occurs because the total recombination dipole goes through a minimum for a certain value of the electron's kinetic energy. This happens because the radial integral for the recombination from $d$ waves changes sign. Moreover, the phase of the dipole varies quickly across the minimum position. This will translate to a similar variation in the harmonic phase that should be observable in experiments using the reconstruction of attosecond beating by interference of two-photon transitions (RABBITT) technique [23,24] or mixed gases [15].

The present example enables us to directly compare the information content of a HHG spectrum with that of a photoelectron experiment. The radial integrals appearing in Eq. (3) are identical for both cases, but a photoionization cross section represents an angle-integrated measurement and is thus described by an incoherent sum over the emitted partial waves whereas HHG is an inherently differential technique which is expressed as a coherent sum over recombining partial waves. This explains why the minimum seen in HHG is much deeper than the minimum seen in photoionization.

The conversion from the electron's kinetic energy $\left(k^{2} / 2\right)$ to the photon energy $(\Omega)$ has been a subject of debate in recent work, some studies using $\Omega=k^{2} / 2$, others 
$\Omega=k^{2} / 2+I_{p}[3,8,25]$. The disagreement originates from the fact that the kinetic energy of the electron is not clearly defined at the moment of recollision because the electron is accelerated by the ionic potential. The use of plane waves and the relation $\Omega=k^{2} / 2+I_{p}$ predicts that the minimum in argon lies around $21 \mathrm{eV}$. If the other dispersion relation is used, the minimum shifts to $5 \mathrm{eV}$, so the agreement with experiment is even worse. In the case of exact continuum functions, the dispersion relation can only be $\Omega=k^{2} / 2+I_{p}$ because the momentum $k$ of the electron is defined at asymptotic distances from the core where the ionic potential is negligible.

The main approximations of the present method lie in neglecting the laser field which can be justified using a semiclassical argument that compares the magnitude of the energies involved in the process. The electron corresponding to the observed minimum position has a kinetic energy of $37 \mathrm{eV}$ and the ionic potential is $15.8 \mathrm{eV}$. However, the variation of the electrostatic potential of the laser field across the region where recombination takes place amounts to a few electron volts only. This means that the effect of the laser field on the electron at the moment of recombination is negligible and suggests that field-free continuum wave functions properly describe the region where recombination occurs.

We have checked the consistency of our results by replacing the ground state wave function obtained from the effective one-electron potential with the wave function obtained from an all-electron quantum chemical ab initio calculation using the Hartree-Fock method and the correlation consistent polarized valence triple-zeta basis set. We have found that the position in the minimum of the recombination dipole is left essentially unchanged. In our method, there is no need to orthogonalize the continuum functions with the bound states [26] because the continuum is orthogonal by construction. A more rigorous treatment of the exchange interaction could be implemented following the method introduced in Ref. [27].

In conclusion, we have observed and characterized a new kind of minimum in high-harmonic generation. As opposed to the well-known two-center interference in diatomic molecules [6] that depends on the geometric structure (and the bonding or antibonding nature of the orbital), the present minimum results solely from the electronic structure and therefore represents a "fingerprint" of the orbital. HHG thus allows the precise measurement of electronic structure. It seems that, at least for atoms, the laser field can be neglected in the description of recombination. This is reminiscent of the recent discovery that the angular distribution of rescattered electrons following laserinduced recollision is in agreement with field-free scattering from the ionic potential [28]. We have shown that the recombination process of HHG represents a differential measurement of the photorecombination cross section.

We have shown that an appropriate description of the recombination dipoles underlying high-harmonic genera- tion requires an accurate description of the continuum. The method can be applied even to polyatomic molecules using quantum chemical $a b$ initio methods [29]. In this perspective, high-harmonic generation gives access to details of the electronic structure of the atom or molecule. This may be exploited to measure the electronic structure of more complex atoms and molecules, possibly in a time-resolved fashion.

One of the authors (H.J.W.) acknowledges financial support from the Swiss National Science Foundation (SNF). P. C. and J. B. gratefully acknowledge research support from the Natural Sciences and Engineering Research Council of Canada and the Canadian Institute for Photonic Innovations.

[1] E. Goulielmakis et al., Science 320, 1614 (2008).

[2] S. Baker et al., Science 312, 424 (2006).

[3] J. Itatani et al., Nature (London) 432, 867 (2004).

[4] P. B. Corkum, Phys. Rev. Lett. 71, 1994 (1993).

[5] H. Niikura et al., Nature (London) 417, 917 (2002).

[6] M. Lein, N. Hay, R. Velotta, J. P. Marangos, and P. L. Knight, Phys. Rev. Lett. 88, 183903 (2002).

[7] T. Kanai, S. Minemoto, and H. Sakai, Nature (London) 435, 470 (2005).

[8] C. Vozzi et al., Phys. Rev. Lett. 95, 153902 (2005).

[9] O. Smirnova, M. Spanner, and M. Ivanov, Phys. Rev. A 77, 033407 (2008).

[10] Z. B. Walters, S. Tonzani, and C. H. Greene, J. Phys. B 40, F277 (2007)

[11] T. Morishita, A.-T. Le, Z. Chen, and C. D. Lin, Phys. Rev. Lett. 100, 013903 (2008).

[12] M. F. Ciappina, C. C. Chirila, and M. Lein, Phys. Rev. A 75, 043405 (2007).

[13] J. W. Cooper, Phys. Rev. 128, 681 (1962).

[14] J. S. Robinson et al., Appl. Phys. B 85, 525 (2006).

[15] T. Kanai, E. J. Takahashi, Y. Nabekawa, and K. Midorikawa, Phys. Rev. Lett. 98, 153904 (2007).

[16] P. Colosimo et al., Nature Phys. 4, 386 (2008).

[17] J. A. R. Samson and W. C. Stolte, J. Electron Spectrosc. Relat. Phenom. 123, 265 (2002).

[18] A. L'Huillier and P. Balcou, Phys. Rev. Lett. 70, 774 (1993).

[19] H. G. Muller, Phys. Rev. A 60, 1341 (1999).

[20] D. J. Tannor, Introduction to Quantum Mechanics: A TimeDependent Perspective (University Science Books, Sausalito, CA, 2007).

[21] L. Young et al., Phys. Rev. Lett. 97, 083601 (2006).

[22] Z. H. Loh et al., Phys. Rev. Lett. 98, 143601 (2007).

[23] P. M. Paul et al., Science 292, 1689 (2001).

[24] Y. Mairesse et al., Science 302, 1540 (2003).

[25] J. Levesque, D. Zeidler, J. P. Marangos, P. B. Corkum, and D. M. Villeneuve, Phys. Rev. Lett. 98, 183903 (2007).

[26] R. Santra and A. Gordon, Phys. Rev. Lett. 96, 073906 (2006).

[27] S. Patchkovskii, Z. Zhao, T. Brabec, and D. M. Villeneuve, Phys. Rev. Lett. 97, 123003 (2006).

[28] D. Ray et al., Phys. Rev. Lett. 100, 143002 (2008).

[29] S. Tonzani, Comput. Phys. Commun. 176, 146 (2007). 